\title{
CUA-CUOG guidelines for the management of castration-resistant prostate cancer (CRPC): 2013 update
}

\author{
Fred Saad, MD, FRCSC; ${ }^{*}$ Sebastien Hotte, MD, FRCSC; ${ }^{\dagger}$ Charles Catton, MD, FRCPC; ${ }^{\xi}$ Darrel Drachenberg, \\ MD, FRCSC, $;$ Antonio Finelli, MD, FRCSC; Neil Fleshner, MD, FRCSC,; Martin Gleave, MD, FRCSC, ${ }_{;}^{\ddagger}$ Anil Ka-

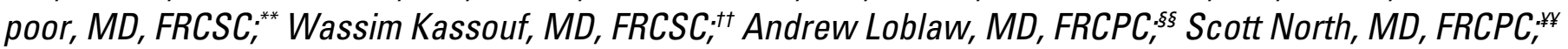 \\ Nawaid Usmani, MD, FRCSC, $;$ Kim N. Chi, MD, FRCSC ${ }^{ \pm \pm}$
}

\begin{abstract}
*Division of Urologic Oncology Centre Hospitalier de I'Université de Montréal, Montréal, QC; '十ivision of Medical Oncology, Associate Professor, McMaster University, Hamilton, ON; ${ }^{\circledR} D$ Department of Radiation Oncology, The Princess Margaret Hospital and University of Toronto, Toronto, ON; *Department of Surgery, Section of Urology, University of Manitoba, St. Boniface General Hospital, Winnipeg, MB; \pm Division of Urology, Department of Oncology, Princess Margaret Hospital, Toronto ON; ' ¿Department of Urologic Sciences, UBC, Vancouver, BC; * ${ }^{*}$ Division of Urology, McMaster University, Hamilton, ON; ${ }^{\dagger+}$ Department of Urology, McGill University Health Centre, Montréal, QC; §§Department of Radiation Oncology, Odette Cancer Centre, Sunnybrook Health Sciences Centre, University of Toronto, Toronto, ON; *\#Department of Oncology, Cross Cancer Institute, Edmonton, AB; ${ }^{ \pm}$British Columbia Cancer Agency, Vancouver, BC
\end{abstract}

Cite as: Can Urol Assoc J 2013:7 (7-8):213-7. hittp://dx.doi.org/10.5489/cuaj.1542 Published online August 19, 2013.

\section{Summary of CRPC guidelines 2013}

Castration-resistant prostate cancer (CRPC) includes a wide range of disease types: from patients without metastases or symptoms with rising prostate-specific antigen (PSA) levels despite androgen deprivation therapy (ADT) to patients with metastases and significant debilitation due to cancer symptoms. The panel recommends that ADT be continued in the presence of CRPC (Fig. 1).

\section{Clinical scenarios and management options for patients with CRPC}

\section{Rising PSA non metastatic CRPC}

There is no standard of care and no approved regimen in MO CRPC. Anti-androgen (AA) therapy should be discontinued if patients are receiving these agents. Secondary hormonal treatments (not including abiraterone or enzalutamide) may be attempted (Level 3, Grade C).

\section{Detection of metastases and imaging}

It is suggested to screen for bone metastases with bone scans and monitor for lymph node and visceral metastases/progression with imaging of the abdomen and chest. Patients with a rapid PSA doubling time (PSADT) $(<8$ months) are at risk for developing earlier metastases. Imaging in these patients should be performed every 3 to 6 months. Patients with slower PSA DT (>12 months) should be screened every 6 to 12 months (Grade C).

\section{Metastatic CRPC without symptoms}

Abiraterone acetate $1000 \mathrm{mg} /$ day plus prednisone $5 \mathrm{mg} /$ twice daily is recommended as first-line therapy (Level 1, Grade A). Abiraterone acetate significantly improved radiographic progression-free survival, time to pain progression and time to chemotherapy initiation; it also delayed ECOG (Eastern Cooperative Oncology Group) performance status deterioration. There was a non-significant 5 -month improvement in overall survival.

Treatment with docetaxel $75 \mathrm{mg} / \mathrm{m}^{2}$ every 3 weeks plus $5 \mathrm{mg}$ oral prednisone twice daily should be offered (Level 1, Grade A). Docetaxel was shown to improve overall survival, disease control, symptom palliation and quality of life.

However, the timing of docetaxel therapy in men with evidence of metastases, but without symptoms, should be discussed with patients; therapy should be individualized based on the patient's clinical status and preference.

AA should be discontinued if patients are on it to test for an AA withdrawal response (AAWD). Introduction of, or changes to, a first-generation AA or the use of corticosteroids with or without ketoconazole may be considered (Level 3 Grade C). 
Saad et al.

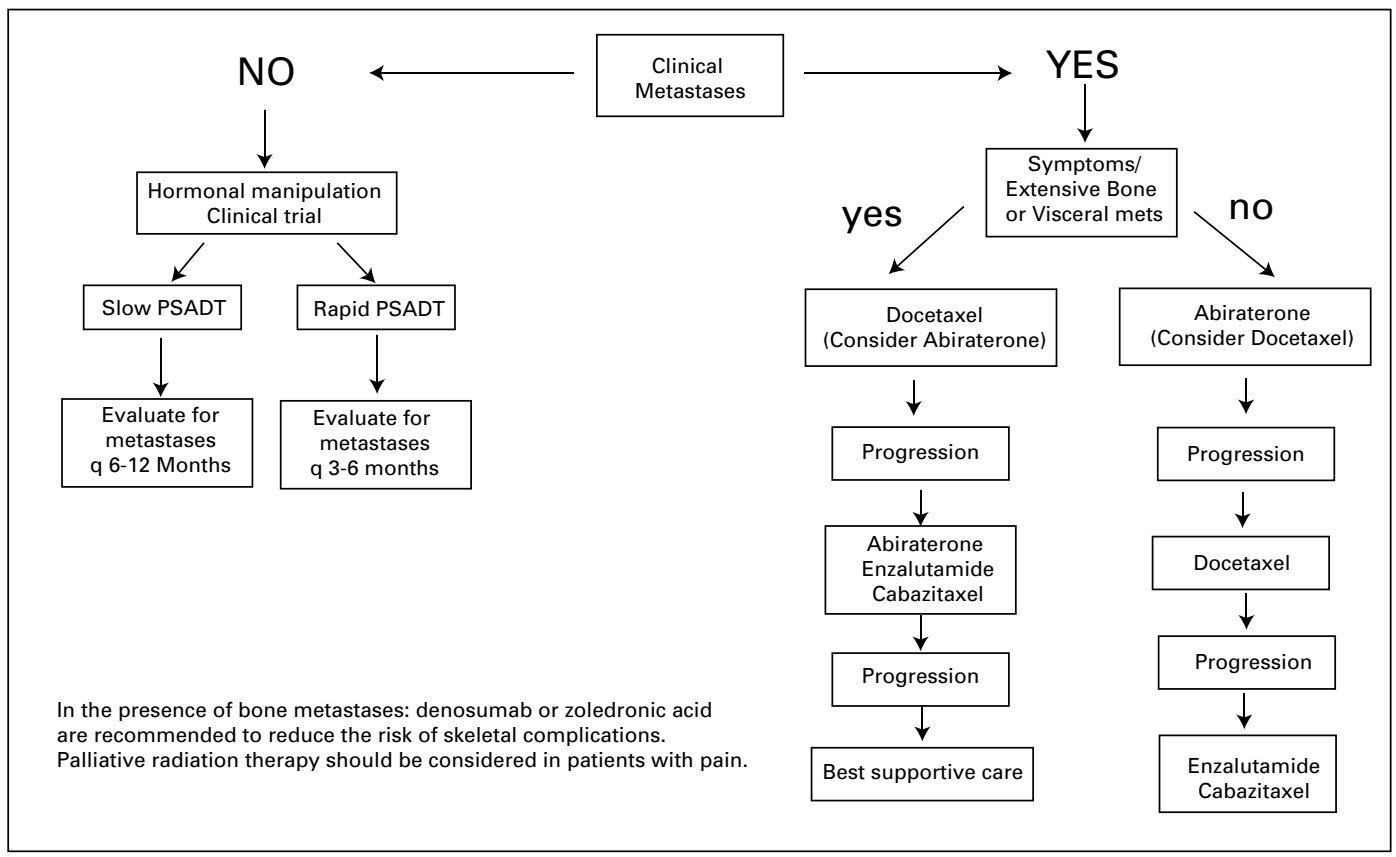

Fig. 1. Management of castration-resistant prostate cancer. PSADT: prostate-specific antigen doubling time.

\section{Metastatic CRPC with symptoms}

Treatment with docetaxel $75 \mathrm{mg} / \mathrm{m}^{2}$ every 3 weeks plus $5 \mathrm{mg}$ oral prednisone twice daily is recommended (Level 1 , Grade A). Docetaxel was shown to improve overall survival, disease control, symptom palliation and quality of life.

Abiraterone acetate $1000 \mathrm{mg}$ /day plus prednisone $5 \mathrm{mg}$ twice daily may be considered as first-line therapy in patients who cannot receive or refuse docetaxel (expert opinion). The study in chemotherapy-naïve patients did not include patients with moderate or severe pain; therefore, the efficacy remains less well-documented in patients with significant symptoms.

\section{Metastatic CRPC who progress after docetaxel-based chemotherapy}

\section{Options with survival benefit}

- Cabazitaxel $\left(25 \mathrm{mg} / \mathrm{m}^{2}\right)$ plus prednisone (5 mg/day) (Level 1, Grade A)

- Abiraterone acetate (1000 mg per day) plus prednisone (5 mg twice daily) (Level 1, Grade A)

- Enzalutamide (160 mg/day) (Level 1, Grade A)

\section{Options with unknown survival benefit}

Docetaxel plus prednisone re-exposure in patients who have had a previous favourable response to docetaxel may be reasonable (expert opinion). Mitoxantrone plus prednisone may be offered for palliative pain relief (Grade C).

\section{Patients with bone metastases}

In men with CRPC and bone metastases, denosumab (120 mg subcutaneous) or zoledronic acid (4 mg intravenous) every 4 weeks, along with daily calcium and vitamin D supplementation, is recommended to prevent disease-related skeletal complications, including pathological fractures, spinal cord compression, surgery or radiation therapy to bone (Level 1, Grade A).

\section{Definition of CRPC}

Castration-resistant prostate cancer (CRPC) is defined by disease progression despite androgen depletion therapy (ADT) and may present as either a continuous rise in serum prostatespecific antigen (PSA) levels, the progression of pre-existing disease, and/or the appearance of new metastases.

Advanced prostate cancer has been known under a few names over the years, including hormone-resistant prostate cancer (HRPC) and androgen-insensitive prostate cancer (AIPC). Most recently, the terms castration-resistant prostate cancer or castration-recurrent prostate cancer were introduced with the realization that extra testicular androgen production plays a significant role in the resistance of prostate cancer cells to medical or surgical castration therapy. ${ }^{1}$

In their second publication, the Prostate Cancer Working Group defined CRPC as a continuum on the basis of whether metastases are detectable (clinically or by imaging) and whether the serum testosterone is in the castrate range by 
surgical orchidectomy or medical therapy. ${ }^{2}$ This definition creates a clinical-states model where patients can be classified. The rising PSA states (castrate and non-castrate) signify that no detectable (measurable or non-measurable) disease has ever been found. The clinical metastases states (castrate and non-castrate) signify that disease was detectable at some point in the past, regardless of whether it is detectable now. ${ }^{3}$

Prognosis is associated with several factors, including performance status, presence of bone pain, extent of disease on bone scan, and serum lactate dehydrogenase and alkaline phosphatase levels. Bone metastases will occur in $90 \%$ of men with CRPC and can produce significant morbidity, including pain, pathologic fractures, spinal cord compression and bone marrow failure. Paraneoplastic effects, including anemia, weight loss, fatigue, hypercoagulability and increased susceptibility to infection, are also common.

CRPC includes patients without metastases or symptoms with rising PSA levels despite ADT to patients with metastases and significant debilitation due to cancer symptoms.

\section{Management of CRPC}

\section{Secondary hormonal manipulations with first-generation non-steroidal antiandrogens}

In patients who develop CRPC and who are asymptomatic or minimally symptomatic, secondary hormonal treatments may be attempted (Level 3, Grade C).

To this date, no study of secondary hormone treatment has shown survival benefits; most trials have been smaller and were not designed to evaluate overall survival and were heavily confounded by future treatments used. In patients treated with luteinizing hormone-releasing hormone (LHRH) agonist/antagonist monotherapy or who have had an orchidectomy, the addition of total androgen blockade (TAB) with androgen receptor antagonists, such as bicalutamide, can offer PSA responses in $30 \%$ to $35 \%$ of patients. ${ }^{4}$

For patients who have undergone $\mathrm{TAB}$, the antiandrogen should be discontinued to test for an antiandrogen withdrawal response (AAWD). Introducing or changing antiandrogen (AA) or using corticosteroids with or without ketoconazole have been noted to cause transient PSA reductions in about $30 \%$ of patients (Level 3 Grade C).

Because the androgen receptor remains active in most patients who have developed castration-resistant disease, it is recommended by groups, such as ASCO (American Society of Clinical Oncology), NCCN (National Comprehensive Cancer Network), CCO (Cancer Care Ontario) and others, that ADT should be continued (Level 3, Grade C).

\section{Non-metastatic CRPC}

There is no standard of care and no approved regimen in MO CRPC. AA therapy should be discontinued if patients are receiving these agents. Secondary hormonal treatments may be attempted (Level 3, Grade C).

\section{Detection of metastases and imaging}

For patients who progress on ADT without evidence of distant metastases, it is suggested to screen for bone metastases with bone scans and monitor for lymph node and visceral metastases/progression with imaging of the abdomen and chest.

Patients with a rapid PSADT ( $<8$ months) are at risk for developing earlier metastases. Imaging in these patients should be performed every 3 to 6 months.

Patients with a slower PSADT (>12 months) should be screened every 6 to 12 months (expert opinion). Imaging techniques most commonly used include nuclear bone scans and abdominal computed tomography and chest X-ray. The role of magnetic resonance imaging and positron-emission tomography are still unclear.

\section{Treatment of metastatic CRPC}

\section{Androgen receptor (AR) signalling therapeutic options}

Novel agents that can affect the androgen receptor signalling have recently been developed and have renewed the enthusiasm for effective hormone manipulation. Currently, in men with CRPC, phase III clinical trials evaluating whether prednisone and abiraterone acetate, a potent and irreversible inhibitor of CYP-17, a critical enzyme in androgen biosynthesis, can improve survival when compared to prednisone and placebo have been published..$^{5-8}$ In the post-docetaxel setting, abiraterone/prednisone was shown to significantly prolong median overall survival compared to placebo/prednisone by 3.9 months to 14.8 versus 10.9 months (hazard ratio $[\mathrm{HR}] 0.64, p=0.0001$ ). Abiraterone acetate was also shown to improve patient-reported outcomes (pain, quality of life) and delay skeletal-related events (SREs). In light of these positive results, abiraterone was approved by Health Canada for CRPC patients previously treated with docetaxel.

Abiraterone: In patients progressing on or after docetaxelbased chemotherapy, abiraterone acetate (1000 mg per day) plus prednisone ( $5 \mathrm{mg}$ twice daily) is an option that should be offered (Level 1, Grade A). 
Saad et al.

The 2012 AFFIRM trial compared enzalutamide (formerly MDV-3100, a potent inhibitor of the androgen receptor) ${ }^{9}$ and placebo in patients previously treated with docetaxel. ${ }^{10}$ The study demonstrated a significant overall survival advantage of 4.8 months (HR 0.62), with improvements in pain, PSA and radiologic progression-free survival (PFS). Enzalutamide has been approved for use by the US FDA and received Health Canada approval in May 2013 as a treatment option for metastatic CRPC following docetaxelbased chemotherapy.

Enzalutamide: In patients progressing on or after docetaxelbased chemotherapy, enzalutamide (160 $\mathrm{mg}$ per day) is an option that should be offered (Level 1, Grade A).

At the 2012 ASCO Annual Meeting, the results of a phase III study with abiraterone acetate in patients that were chemotherapy naïve was presented by Ryan and colleagues. ${ }^{8}$ The study was unblinded at the second interim analysis due to a very significant improvement in radiographic PFS, and patients on the placebo arm crossed over to active therapy. The co-primary endpoint of overall survival showed a strong trend towards statistical significance. Other endpoints were an improvement in time to chemotherapy and opiate use. The results of this study have now been published ${ }^{8}$ and the US FDA has recently expanded the use of abiraterone acetate to include patients who are chemotherapy naïve. This indication has received Health Canada approval in May 2013.

Abiraterone acetetate $1000 \mathrm{mg} /$ day plus prednisone $5 \mathrm{mg}$ twice daily is recommended as first-line therapy for metastatic CRPC (Level 1, Grade A). In asymptomatic or minimally symptomatic patients, abiraterone acetate significantly improved radiographic PFS. Abiraterone also significantly delayed time to pain progression, time to chemotherapy initiation, and deterioration of the ECOG performance status. There was a non-significant 5-month improvement in overall survival.

We await the results of a similar trial with enzalutamide in chemotherapy-naïve CRPC patients (PREVAIL). The updated NCCN guidelines for 2013 have added both abiraterone and enzalutamide as first-line therapeutic options for metastatic CRPC. Abiraterone and enzalutamide may be potential therapeutic options in patients who are deemed chemotherapy ineligible. The use of these agents prior to docetaxel may be limited depending on provincial reimbursement guidelines.

\section{Systemic corticosteroid therapy}

Corticosteroid therapy with low dose prednisone or dexamethasone may also offer improvements in PSA values and/ or palliative outcomes in up to $30 \%$ of patients in both symptomatic and asymptomatic men. Steroids may also exert an anti-neoplastic effect on prostate cancer ${ }^{11,12}$ (Level 3, Grade C).

\section{First-line systemic chemotherapy}

Currently, only patients with CRPC who have detectable macroscopic metastatic disease should be considered for systemic chemotherapy outside of a clinical trial. Patients with advanced prostate cancer should be referred early for possible chemotherapy and should optimally receive multidisciplinary care to maximize survival and optimize quality of life. Because any treatment for advanced disease remains non-curative, patients with advanced prostate cancer should be encouraged to participate in clinical trials.

Tannock and colleagues randomized 1006 patients to one of three treatment arms: (1) docetaxel $\left(75 \mathrm{mg} / \mathrm{m}^{2}\right.$ intravenous [IV], every 3 weeks); (2) docetaxel $\left(30 \mathrm{mg} / \mathrm{m}^{2}, 5\right.$ times weekly for 5 of 6 weeks), or (3) control therapy with mitoxantrone. ${ }^{13}$ The study reported improved survival with docetaxel (every 3 weeks) compared with mitoxantrone-prednisone (median survival: 18.9 vs. 16.5 months; HR 0.76 [95\% confidence interval $(\mathrm{Cl}), 0.62-0.94])$, two-sided $p=0.009)$. No overall survival benefit was observed with docetaxel given on a weekly schedule (HR 0.91, [95\% Cl, 0.75-1.11], two-sided $p=0.36)$. Significantly more patients treated with docetaxel (every 3 weeks) achieved a pain response compared with patients receiving mitoxantrone (35\% vs. $22 \%, p=0.01)$. Quality of life response, defined as a sustained 16-point or greater improvement from baseline on two consecutive measurements, was higher with docetaxel given every 3 weeks $(22 \%$ vs. $13 \%, p=0.009)$ or weekly $(23 \%$ vs. $13 \%$, $p=0.005)$ compared with mitoxantrone. PSA response rates were also statistically significantly higher with docetaxel compared to mitoxantrone. In the 2 trials, $27 \%(n=412)$ and $29 \%(n=196)$ of patients had measurable disease.

\footnotetext{
Docetaxel: Based on the results of randomized controlled trials, it is recommended that for men with metastatic CRPC and clinical or biochemical evidence of progression, treatment with docetaxel $75 \mathrm{mg} / \mathrm{m}^{2}$ IV every 3 weeks with $5 \mathrm{mg}$ oral prednisone twice daily should be offered to improve overall survival, disease control, symptom palliation and quality of life ${ }^{13,14}$ (Level 1, Grade A).
}

Although patients received up to 10 cycles of treatment if no progression and no prohibitive toxicities were noted, the duration of therapy should be based on the assessment of benefit and toxicities. Rising PSA only should not be used as the sole criteria for progression; assessment of response should incorporate clinical and radiographic criteria. Alternative therapies that have not demonstrated improvement in overall survival, but can provide disease control, palliation and improve quality of life, include 
weekly docetaxel plus prednisone, and mitoxantrone plus prednisone ${ }^{15}$ (Level 2, Grade B).

The timing of docetaxel therapy in men with evidence of metastases, but without symptoms, should be discussed with patients and therapy should be individualized based on patients' clinical status and preferences (Level 3, Grade C).

Use of estramustine in combination with other cytotoxic agents is not recommended due to the increased risk of clinically important toxicities. There is no evidence to support the use of this combination to improve survival or palliation (Level 2, Grade C).

For patients who do not respond to first-line ADT or who progress clinically or radiologically without significant PSA elevations may have neuroendocrine differentiation. Biopsy of accessible lesions should be considered to identify these patients; these patients should then be treated with combination chemotherapy, such as cisplatin/etoposide or carboplatin/etoposide (Level 3, Grade C).

\section{Second-line systemic chemotherapy}

Mitoxantrone: Until recently mitoxantrone was considered de facto second-line chemotherapy, but it has limited activity and increased toxicity in this setting (Level 4, Grade D).

For patients who have not demonstrated definitive evidence of resistance to docetaxel, re-treatment with docetaxel can be considered ${ }^{16-18}$ (Level 3, Grade C).

Recent results comparing cabazitaxel to mitoxantrone in patients previously treated with docetaxel has shown a statistically significant survival advantage. ${ }^{19}$ This randomized, placebo-controlled trial recruited 755 docetaxel-pretreated CRPC patients. Overall survival was the primary endpoint of the study. Patients were randomized to receive prednisone $10 \mathrm{mg} /$ day with 3 -weekly mitoxantrone $12 \mathrm{mg} / \mathrm{m}^{2}$ or cabazitaxel $25 \mathrm{mg} / \mathrm{m}^{2}$. An advantage in survival emerged in favour of the cabazitaxel group, with a median survival of 15.1 months compared with 12.7 months in the mitoxantrone group (HR 0.70; 95\% Cl 0.59, 0.83; $p<0.0001)$.

In light of these positive results, cabazitaxel is considered a therapeutic option for metastatic CRPC patients progressing on or following docetaxel (Level 1, Grade A).

\section{Palliative radiation}

Bone metastases from prostate cancer are often radiosensitive and most men will experience partial or complete pain relief from external beam radiation to a specific lesion. Studies have shown that a single fraction is as effective as 5 or more fractions in providing palliation. However, more patients require retreatment for pain recurrence with single fraction radiation (Level 2, Grade B).

Radionuclide therapy, in the form of systemic strontium-89 therapy, may be useful in the palliation of CRPC when multiple skeletal sites are involved in carefully selected patients. Risks include severe prolonged myelosuppression and transfusion dependence. Strontium-89 may be associated with a worse overall survival as compared to external beam radiotherapy. ${ }^{20,21}$

\section{Bone-targeted therapy}

Bone loss associated with ADT has been shown to increase the risk of fracture. ${ }^{22-26}$ Moreover, about $90 \%$ of patients with metastatic CRPC will develop bone metastases, which cause local decreases in bone integrity. Patients are at significant risk of SREs that include pathological fractures, debilitating bone pain requiring palliative radiation therapy and spinal cord compression. Quality of life is affected by these complications.

\section{Denosumab and zoledronic acid: In men with CRPC and bone metastases, denosumab (120 mg subcutaneous [SC]) or zoled- ronic acid (4 mg IV) every 4 weeks is recommended to prevent disease-related SREs, including pathological fractures, spinal cord compression, surgery or radiation therapy to bone $\mathrm{e}^{27,28}$ (Level 1, Grade A).}

Bisphosphonates other than zoledronic acid are not known to be effective to prevent disease-related SREs. Serum creatinine monitoring is suggested prior to each dose of zoledronic acid. Results from the Zoledronic Acid Prostate Cancer Study Group showed fewer men receiving zoledronic acid had SREs than men in the placebo group (38\% vs. $49 \% p=0.02) .{ }^{27}$ Zoledronic acid also increased the median time to first SRE (488 vs. 321 days, $p=0.01$ ). There was an overall $36 \%$ reduction in the rate of SREs in treated patients. Zoledronic acid should be initiated at reduced dose in men with impaired renal function (estimated creatinine clearance $30-60 \mathrm{~mL} / \mathrm{min}$ ). Treatment is not recommended for men with baseline creatinine clearance $<30 \mathrm{~mL} / \mathrm{min}$. Based on recommendations for other settings, bisphosphonate therapy for bone metastases should be continued for as long as clinically beneficial. The optimal duration of zoledronic acid in men with CRPC and bone metastases is undefined; however, efficacy and safety for up to 24 months has been shown.

Other bone-targeted agents include denosumab, a fully humanized monoclonal antibody against RANK ligand. It has been shown to be effective in preventing bone loss and new vertebral fractures due to ADT. ${ }^{26}$ In the setting of metastatic CRPC, denosumab (120 mg SC, every 4 weeks) compared to zoledronic acid ( $4 \mathrm{mg} I \mathrm{~V}$, every 4 weeks) has shown significant improvement in the time to the first SRE (20.7 vs. 17.1 months; $p<0.001$ for non-inferiority; $p=0.008$ for superiority), while overall survival and PFS were not different. ${ }^{28}$ Hypocalcemia was more common in the denosumab arm $(13 \%)$ than in the zoledronic acid arm $(6 \%)(p<0.0001)$ and a non-significant trend toward higher osteonecrosis of the jaw $(\mathrm{ONJ})$ was seen in the denosumab arm $(2.3 \%$ vs 
Saad et al.

$1.3 \% ; p=0.09)$. No dose modification for renal function is necessary in the case of denosumab; however, the risk of hypocalcaemia is increased and calcium monitoring and supplementation is recommended for both denosumab and zoeldronic acid.

Zoledronic acid and denosumab are associated with a $1 \%$ to $2 \%$ risk of ONJ. ${ }^{28}$ Most, but not all, patients who develop ONJ have pre-existing dental problems. Good oral hygiene, baseline dental evaluation for high-risk individuals and avoidance of invasive dental surgery during therapy are recommended to reduce risk of ONJ ${ }^{29-31}$ (Level 3, Grade C). Zoledronic acid and denosumab have been used safely with a variety of cytotoxic chemotherapies in clinical trials. ${ }^{27,28}$

In a recent placebo controlled trial, denosumab significantly increased the bone-metastasis-free survival in patients with non-metastatic CRPC by a median of 4.2 months compared with placebo (29.5 vs. 25.2 months; HR, 0.85; 95\% $\mathrm{Cl}, 0.73-0.98 ; p=0.028) .^{32}$ Denosumab is not approved for bone metastases prevention by Health Canada.

\section{Future potential therapies}

A recently completed phase III study of patients with metastatic CRPC were randomized on a 2:1 basis to either radium-223 chloride (an alpha emitting calcium mimic which preferentially targets bone mestastes) or placebo. Overall survival was the primary endpoint. Median survival was 14 months for the treated patients as opposed to 11.2 months for those who received placebo, conferring an approximate $30 \%$ improvement in overall survival (HR $0.699, p=0.0022)$. This is still unpublished and was presented at ASCO GU in 2012 by Parker and colleagues. ${ }^{33}$

\section{Conclusion}

Advances in treatment for men with CRPC have improved survival and quality of life, but most, if not all, patients eventually succumb from their disease and better treatments are required. Several new agents are being studied in a pre-chemotherapy setting, in combination with docetaxel as well as in the post docetaxel setting. ${ }^{10,29-31,34-36}$ It is hoped that soon we will have more therapeutic options for patients with CRPC. Because CRPC remains an incurable and ultimately fatal illness, inclusion of patients in clinical trials at all stages of the disease remains paramount.

\section{Summary}

For patients with metastatic CRPC, abiraterone acetate and docetaxel-based chemotherapy are approved first-line treatment options in patients with metastatic CRPC. In addition, bone-targeted agents should be used to reduce the risk of SREs. Post-docetaxel therapeutic options with demonstrat- ed survival advantages are now available in Canada and include cabazitaxel, abiraterone and enzalutamide. Other treatments have reported positive results or are presently under investigation and may soon add to the available therapeutic options in Canada.

Acknowledgements: MEDLINE search of the English language and conference proceedings were used to produce the present document. Authors used levels of evidence and grades of recommendation employing the International Consultation on Urologic Disease (ICUD)/WHO modified Oxford Center for Evidence-Based Medicine grading system.

Competing interests: None declared.

This paper has been peer-reviewed.

\section{References}

1. Mohler $\mathrm{JL}$, Gregory $\mathrm{CW}$, Ford $\mathrm{OH} 3 \mathrm{rd}$, et al. The androgen axis in recurrent prostate cancer. Clin Cancer Res 2004;10:440. http://dx.doi.org/10.1158/1078-0432.CCR-1146-03

2. Scher HI, Halabi S, Tannock I, et al. Design and end points of clinical trials for patients with progressive prostate cancer and castrate levels of testosterone: recommendations of the Prostate Cancer Clinical Trials Working Group. J Clin Oncol 2008;26:1148-59. http://dx.doi.org/10.1200/JC0.2007.12.4487

3. Smith MR, Kabbinavar F, Saad F, et al. Natural history of rising serum prostate-specific antigen in men with castrate nonmetastatic prostate cancer. J Clin Oncol 2005;23:2918-25. http://dx.doi.org/10.1200/ IC0.2005.01.529

4. Small EJ, Halabi S, Dawson NA, et al., Antiandrogen withdrawal alone or in combination with ketoconazole in androgen-independent prostate cancer patients: a phase III trial (CALGB 9583), J Clin Oncol 2004;22:1025-33. http://dx.doi.org/10.1200/JC0.2004.06.037

5. Hartmann $R$, Ehmer $P$, Haidar $S$, et al., Inhibition of CYP 17 , a new strategy for treatment of prostate cancer. Arch Pharm 2002;335:119-28. http://dx.doi.org/10.1002/1521-4184(200204)335:4<119::AIDARDP119>3.0.C0;2\#

6. de Bono JS, Logothetis $\mathrm{CJ}$, Molina A, et al; and the COU-AA-301 Investigators. Abiraterone and increased survival in metastatic prostate cancer. N Engl J Med 2011;364:1995-2005. http://dx.doi. org/10.1056/NEJMoa1014618

7. Fizazi K, Scher HI, Molina A; and the COU-AA-301 Investigators. Abiraterone acetate for treatment of metastatic castration-resistant prostate cancer: final overall survival analysis of the COU-AA-301 randomised,double-blind, placebo-controlled phase 3 study. Lancet Oncol 2012;13:983-92. http:// dx.doi.org/10.1016/S1470-2045(12)70379-0

8. Ryan CJ, Smith MR, de Bono IS, et al; and the COU-AA-302 Investigators. Abiraterone in Metastatic Prostate Cancer without Previous Chemotherapy. N Engl J Med 2013;368:138-48. 2012 Dec 10 [Epub ahead of print].

9. Tran C, Ouk S, Clegg NJ, et al. Development of a second-generation antiandrogen for treatment of advanced prostate cancer. Science 2009;324:787. http://dx.doi.org/10.1126/science.1168175

10. Scher HI, Fizazi K, Saad F, et al; and the AFFIRM Investigators. Increased survival with enzalutamide in prostate cancer after chemotherapy. N Engl J Med 2012;367(13):1187-97. http://dx.doi. org/10.1056/NEJMoal207506

11. Storlie JA, Buckner JC, Wiseman GA, et al., Prostate specific antigen levels and clinical response to low dose dexamethasone for hormone-refractory metastatic prostate carcinoma. Cancer 1995;76:96-100. http://dx.doi.org/10.1002/1097-0142(19950701)76:1<96::AID-CNCR2820760114>3.0.C0;2-E

12. Heng DY, Chi KN. Prednisone monotherapy in asymptomatic hormone refractory prostate cancer. Can J Urol 2006; $13: 3335-9$

13. Tannock IF, de Wit R, Berry WR, et al., Docetaxel plus prednisone or mitoxantrone plus prednisone for advanced prostate cancer, N Engl J Med 2004;351:1502-12. http://dx.doi.org/10.1056/ NEJMoa040720

14. Petrylak DP, Tangen CM, Hussain MHA, et al., Docetaxel and estramustine compared with mitoxantrone and prednisone for advanced refractory prostate cancer. N Engl J Med 2004;351:1513-20. http:// dx.doi.org/10.1056/NEJMoa041318 
15. Michels J, Montemurro T, Murray N, et al. First- and second-line chemotherapy with docetaxel or mitoxantrone in patients with hormone-refractory prostate cancer: does sequence matter? Cancer 2006;106:10416. http://dx.doi.org/10.1002/cncr.21695

16. Oh WK, Manola J, Babcic V, et al. Response to second-line chemotherapy in patients with hormone refractory prostate cancer receiving two sequences of mitoxantrone and taxanes. Urology 2006;67:1235-40. http://dx.doi.org/10.1016/i.urology.2006.01.006

17. Jankovic B, Beardsley E, Chi KN. Rechallenge with docetaxel as second-line chemotherapy in patients with metastatic hormone refractory prostate cancer (HRPC) after previous docetaxel: a population based analysis [abstract \#196]. 2008 ASCO Genitourinary Symposium; 2008.

18. Eymard J, Oudard S, Gravis $G$, et al. Docetaxel reintroduction in patients with metastatic castration-resistant docetaxel-sensitive prostate cancer: a retrospective multicentre study. BJU Int 2010 Mar 9. http://dx.doi. org/10.1111/i.1464-410X.2010.09296.x

19. de Bono JS, Oudard S, Ozguroglu M, et al. Prednisone plus cabazitaxel or mitoxantrone for metastatic castration-resistant prostate cancer progressing after docetaxel treatment: a randomised open-label trial. Lancet 2010;376:1147. http://dx.doi.org/10.1016/S0140-6736(10)61389-X

20. Porter AT, MCEwan AJ. Strontium-89 as an adjuvant to external beam radiation improves pain relief and delays disease progression in advanced prostate cancer: results of a randomized controlled trial. Semin Oncol 1993;20:38-43.

21. Oosterhof G0, Roberts JT, de Reijke TM, et al. Strontium(89) chloride versus palliative local field radiotherapy in patients with hormonal escaped prostate cancer: a phase III study of the European Organisation for Research and Treatment of Cancer, Genitourinary Group. Eur Urol 2003;44:519-26. http://dx.doi. org/10.1016/S0302-2838(03)00364-6

22. Diamond TH, Higano CS, Smith MR, et al. Osteoporosis in men with prostate carcinoma receiving androgendeprivation therapy: recommendations for diagnosis and therapies. Cancer 2004;100:892. http:// dx.doi.org/10.1002/cncr.20056

23. Shahinian VB, Kuo YF, Freeman JL, et al. Risk of fracture after androgen deprivation for prostate cancer. N Engl J Med 2005;352:154-64. http://dx.doi.org/10.1056/NEJMoa041943

24. Smith MR, McGovern FJ, Zietman AL, et al. Pamidronate to prevent bone loss during androgen-deprivation therapy for prostate cancer. N Engl J Med 2001;345:948-55. http://dx.doi.org/10.1056/ NEJMoa010845

25. Smith MR, Eastham J, Gleason DM, et al., Randomized controlled trial of zoledronic acid to prevent bone loss in men receiving androgen deprivation therapy for nonmetastatic prostate cancer. J Urol 2003;169:2008-12. http://dx.doi.org/10.1097/01.ju.0000063820.94994.95

26. Smith MR, Egerdie B, Hernandez Toriz N, et al. Denosumab in men receiving androgen-deprivation therapy for prostate cancer. N Engl J Med 2009;361:745-55. http://dx.doi.org/10.1056/NEJMo00809003
27. Saad F, Gleason DM, Murray R, et al., Long-term efficacy of zoledronic acid for the prevention of skeletal complications in patients with metastatic hormone refractory prostate cancer. J Natl Cancer Inst 2004;96:879-82. http://dx.doi.org/10.1093/inci/dihl41

28. Fizazi K, Carducci M, Smith M, et al. Denosumab versus zoledronic acid for treatment of bone metastases in men with castration-resistant prostate cancer: a randomised, double-blind study. Lancet 201 1;377:813. http://dx.doi.org/10.1016/S0140-6736(10)62344-6

29. Dreicer R, Agus DB, MacVicar GR, et al. Safety, pharmacokinetics, and efficacy of TAK-700 in metastatic castration-resistant prostate cancer: A phase I/II, open-label study. J Clin Oncol 2010;28 (Suppl 15: abstr 3084).

30. Kantoff PW, Schuetz TJ, Blumenstein BA, et al. Overall survival analysis of a phase II randomized controlled trial of a Poxviral-based PSA-targeted immunotherapy in metastatic castration-resistant prostate cancer. J Clin Oncol 2010;28:1099. http://dx.doi.org/10.1200/JC0.2009.25.0597

31. Saad F, Hotte SJ, North SA, et al. A phase II randomized study of custirsen (OGX-011) combination therapy in patients with poor-risk hormone refractory prostate cancer who relapsed on or within six months of 1st-line docetaxel therapy. Can Urol Assoc J 2008;2:568.

32. Smith MR, Saad F, Coleman R, et al. Denosumab and bone-metastasis-free survival in men with castrationresistant prostate cancer: results of a phase 3, randomised, placebo-controlled trial. Lancet 2012;379:3946. http://dx.doi.org/10.1016/50140-6736(11)61226-9

33. Parker C, Nilsson S, Heinrich D, et al. Updated analysis of the phase III, double-blind, randomized, multinational study of radium-223 chloride in castration-resistant prostate cancer (CRPC) patients with bone metastases (ALSYMPCA) [abstract]. J Clin Oncol 2012;30: (suppl; abstr LBA4512).

34. Saad F. Src as a therapeutic target in men with prostate cancer and bone metastases. BJU Int 2009;103:434. http://dx.doi.org/10.1111/j.1464-410X.2008.08249.x

35. Araujo J, Armstrong AJ, Braud EL, et al. Dasatinib and docetaxel combination treatment for patients with castration-resistant progressive prostate cancer: A phase I/II study (CA180086). J Clin Oncol 2009;27.

36. Chi KN, Hotte IS, YU EY, et al. Randomized Phase II Study of Docetaxel and Prednisone With or Without OGX-011 in Patients With Metastatic Castration-Resistant Prostate Cancer. J Clin Oncol 2010:4247. http://dx.doi.org/10.1200/JC0.2009.26.8771

Correspondence: Dr. Fred Saad, Université de Montréal, Directeur, Urologie-Oncologie, CHUM, 1560 Sherbrooke E., Montréal, QC H2L 4M1; fredsaad@videotron.ca; and Dr. Antonio Finelli, Princess Margaret Hospital, 3rd Floor Rm. 130, 610 University Ave., Toronto, ON M5G 2M9; antonio.finelli@uhn.co 